\title{
Caracterización de fincas cacaoteras de la Intercuenca Alto Marañón III, en los distritos de Jaén y Bellavista, Cajamarca
}

\section{Characterization of the cocoa farms of the Alto Marañón III Inter-basin, in the districts of Jaén and Bellavista, Cajamarca}

\author{
Fiorela Ramirez ${ }^{1}$ (D), Santos Herrera ${ }^{1}$ (D), Magali García ${ }^{2}$ (D), Omar Zeballos ${ }^{3 *}(\mathbb{D}$
}

\section{RESUMEN}

La investigación se realizó en dos zonas pertenecientes a la Intercuenca Alto Marañón III, los distritos de Jaén y Bellavista, de la provincia de Jaén, Cajamarca con el objetivo de caracterizar las fincas productoras de cacao. El trabajo se desarrolló con productores pertenecientes a las dos zonas, para la zona de Jaén con una población de 63 productores de la que se tomó una muestra de 39 productores, mientras que para la zona de Bellavista con una población de 314 productores se tomó una muestra de 76. Se realizó una encuesta con aspectos generales, socioeconómicos y factores ambientales, apoyándose con información de observación directa en el predio. Como resultado de esta caracterización se obtuvo que las fincas cacaoteras tienen una amplia complejidad y las familias tienen una dependencia de media a baja con respecto a este cultivo, un grupo representativo de productores incluye otros cultivos dentro o fuera de las fincas de cacao, lo cual les provee de ingresos adicionales para las familias. La evaluación de las fincas, permitió obtener datos claros de las características de forma general y por grupos, 3 grupos para la zona de Jaén y 4 grupos para la zona de Bellavista, los cuales tienen características similares dentro de cada grupo.

Palabras claves: caracterización, indicadores de sustentabilidad, fincas cacaoteras.

\begin{abstract}
The research was carried out in two areas belonging to the Alto Marañón III Interbasin, the districts of Jaén and Bellavista, in the province of Jaén, Cajamarca, with the aim of characterizing cocoa-producing farms. The work was developed with producers belonging to the two zones, for the zone of Jaén with a population of 63 producers from which a sample of 39 producers was taken, while for the zone of Bellavista with a population of 314 producers a sample of 76 was taken. A survey with general, socioeconomic aspects and environmental factors was carried out, supported with information of direct observation in the property. As a result of this characterization, it was obtained that cocoa farms have a wide complexity and families have a medium to low dependence on this crop. A representative group of producers includes other crops within or outside the cocoa farms, which provides additional income for families. The evaluation of the farms, allowed to obtain clear data of the characteristics in general and by groups, 3 groups for the zone of Jaén and 4 groups for the zone of Bellavista, which have similar characteristics within each group.
\end{abstract}

Keywords: characterization, sustainability indicators, cocoa farms.

\footnotetext{
${ }^{1}$ Universidad Nacional de Jaén, Jaén, Perú

${ }^{2}$ Universidad Nacional Toribio Rodríguez de Mendoza de Amazonas, Chachapoyas, Perú

${ }^{3}$ Universidad Católica de Santa María, Escuela de Posgrado, Arequipa, Perú

"Autor de correspondencia.E-mail: omar_zc@hotmail.com
} 


\section{INTRODUCCIÓN}

El cacao (Theobroma cacao L.) es un cultivo cuyo origen se sitúa en América del Sur, abarcando a los países de Perú, Ecuador, Colombia, Brasil, Venezuela (Guerrero, 2007), del fruto de esta planta se obtiene las semillas o almendras de cacao que se utilizan principalmente en la elaboración de chocolates. La producción peruana de cacao para 2018 fue de 134676 mil toneladas de cacao en grano seco la cual está distribuida en 16 regiones, 57 provincias y 259 distritos donde más del $85 \%$ de la producción de cacao se concentra en las regiones de San Martín (40,9\%), Junín (18,3\%), Ucayali (12,3\%), Huánuco (7,7\%) y Cusco $(6,1 \%)$ (Saldaña, 2019).

En cuanto al rendimiento promedio del cacao por regiones, en el 2015 fue de 720 kilogramos por hectárea. Entre las regiones que destacan por su elevada productividad tenemos a la región Pasco, con 1154 kilogramos por hectárea, le sigue Cajamarca, con 1059 kilogramos por hectárea: aunque esta situación no refleja su participación en la producción a nivel nacional, donde apenas representan el $1 \%$ cada uno (MINAGRI, 2016).

El estudio de las fincas y sus agroecosistemas, permite comprender los efectos de la intervención del hombre sobre el ecosistema (Altieri et al., 2011), explica las estrategias campesinas de sobrevivencia frente a la crisis de la agricultura (López et al., 2007) y permite el desarrollo de propuestas que beneficien a los agricultores de Jaén y Bellavista.

La diversidad de fincas hace que sea necesario realizar una caracterización de ellas, como paso previo a cualquier estudio posterior. Conociéndose que la gran heterogeneidad que existe entre las fincas dificulta la toma de decisiones, en ese sentido al agrupar las fincas acorde a sus principales diferencias y relaciones, busca maximizar la homogeneidad dentro del grupo y la heterogeneidad entre estos (Cabrera et al., 2004).

La caracterización es una etapa determinante para el desarrollo del método de investigación en sistemas de producción, y consiste en determinar un conjunto de variables, que distinguen a una zona o unidad de pro- ducción en particular y que la hace diferente a otras (Malagon y Praguer, 2001). Por lo tanto, se considera como una etapa determinante en la investigación de sistemas agrarios (León-Velarde y Quiroz, 1994; FAO y USAC, 1995)

Para caracterizar sistemas agrícolas, se han utilizado diversas técnicas de análisis estadísticos. Así tenemos, que el análisis de varianza es propuesto por Casas y Veitía (2008). Sin embargo, el análisis multivariado, y por ende el análisis clúster y de conglomerados es sugerido por Macedo et al., (2003) y Vargas et al., (2013). Al respecto diversas investigaciones realizadas en Perú sugieren este último, dentro de ellas tenemos a Merma y Julca (2012), quienes caracterizaron lo sistemas agrícolas en la Convención, Cusco. Tuesta et al. (2014) caracterizo fincas cacaoteras en San Martin mientras que Collantes et al. (2015) caracterizaron fincas productoras de mandarinas en Cañete. A nivel internacional se ha reportado esta metodología por Santistevan et al. (2014) quien caracterizo fincas cafetaleras y Anzules et al. (2018) quien caracterizo fincas cacaoteras, ambas estudios realizados en Ecuador.

Bajo este contexto, es que esta investigación es necesaria debido a que existe una carencia frecuente en planes y proyectos de desarrollo rural en cuanto a conocimiento de los sistemas agrícolas. Muchas tecnologías no son adoptadas por los productores, porque no se adecúan a sus problemas más sentidos ni a sus objetivos. Por lo que antes de generar y difundir alternativas tecnológicas, es necesario evaluar los sistemas productivos, siendo el objetivo propuesto de esta investigación el de caracterizar las fincas cacaoteras de la Intercuenca del Alto Marañón III.

\section{MATERIALES Y MÉTODOS}

\section{Descripción del área de estudio}

El presente estudio se realizará en el área localizada en los distritos de Jaén y Bellavista, provincia Jaén, departamento Cajamarca, de la Intercuenca Alto Marañón III (Figura 1). 


\begin{tabular}{|l|}
\hline FECHA : febrero 2018 \\
\hline Datum: WGS 84 \\
\hline Proyeccion: UTM zona 185 \\
\hline Resion: Cajamarca y Amazonas \\
\hline Provincias: Iaen y Utcubamba \\
\hline
\end{tabular}

\section{LEYENDA}
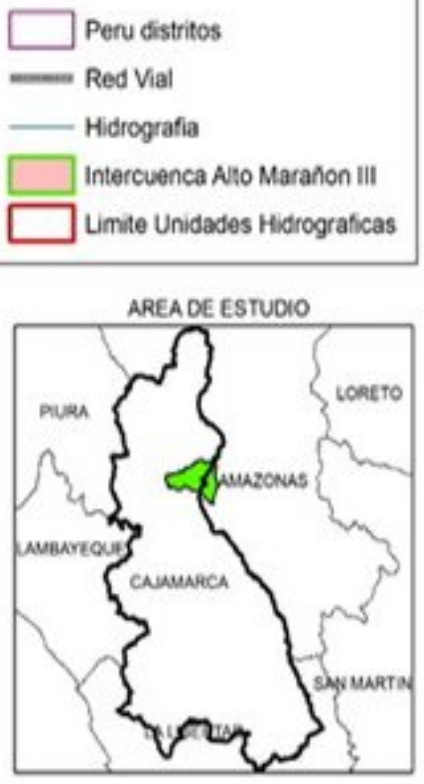

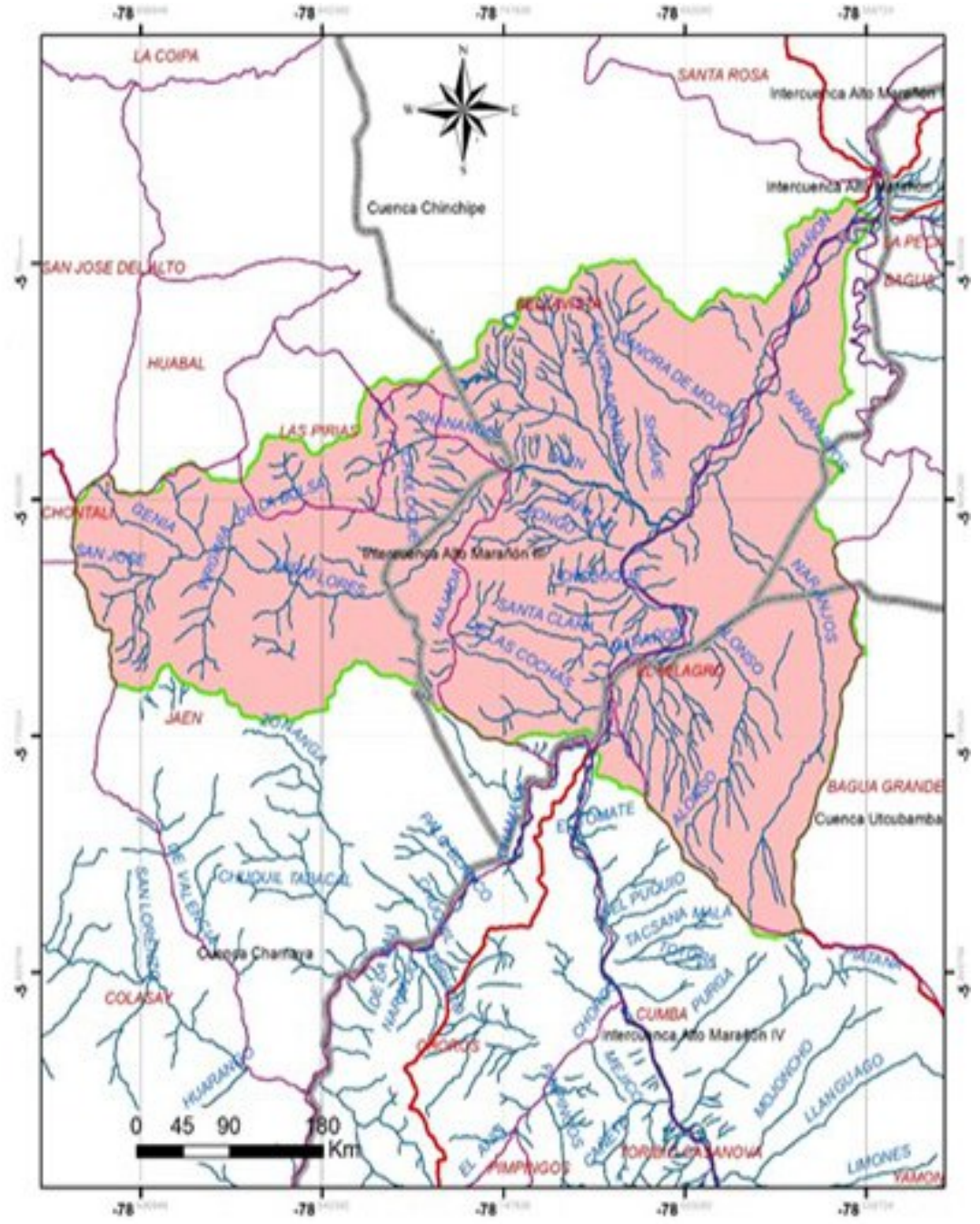

Figura 1. Mapa de localización de la Intercuenca Alto Marañón III.

\section{Metodología}

Se aplicaron cuestionarios en campo y se recolectaron datos directos de finca, con los responsables de la respectiva muestra para caracterizar fincas. Para determinar el tamaño de la muestra, se usó a la fórmula de Scheaffer et al. (1987). Se trabajó con una muestra de 76 productores del distrito de Bellavista, y 39 productores del distrito de Jaén, pertenecientes a la Intercuenca Alto Marañón III. Todos fueron seleccionados aleatoriamente de un padrón general, obtenido de diversas entidades agrarias, como la Junta de Usuarios de Agua, comisiones de regantes, Agroideas, entre otras.

\section{Selección de la muestra}

La muestra fue seleccionada mediante el método de muestra aleatoria simple utilizando para ello el soft- ware Minitab 17, del padrón total de productores cacaoteros de cada distrito.

\section{Análisis de resultados}

Se realizó un análisis multivariado de conglomerados (dendograma) por el método Ward con distancia euclidiana cuadrada a fin de caracterizar las fincas cacaoteras, acorde a las dimensiones de sustentabilidad. Para ello se utilizó el software Minitab 17.

\section{RESULTADOS Y DISCUSIÓN}

\section{Caracterización zona de Jaén}

Se logró determinar para la zona de Jaén 3 grupos de fincas cacaoteras (Figura 2)

La totalidad de los agricultores no pertenece a ninguna asociación, los fundos en su totalidad tienen una extensión inferior a 4 ha, reportándose que tienen acceso a agua, que realizan agricultura convencional y 


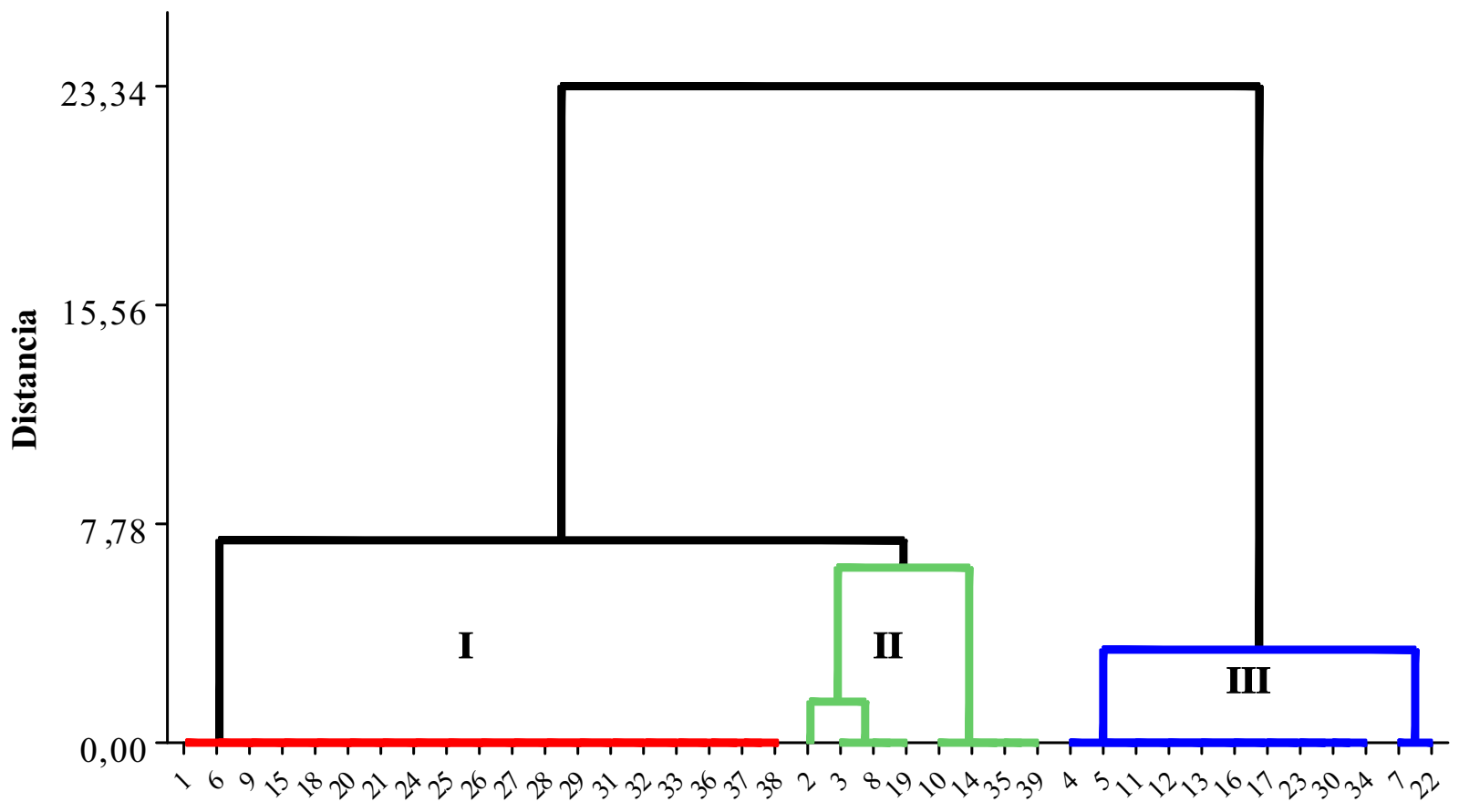

Observaciones

Figura 2. Dendrograma formado por los grupos de fincas cacaoteras para la zona de Jaén

tienen acceso al agua.

El grupo I, es el grupo mayoritario que representa el $48,72 \%$ de la zona de estudio. Entre sus características, resaltó que las fincas cacaoteras poseen como propietarios en su totalidad a varones con instrucción, de los cuales el 52,63\% tiene más de 61 años. Trabajan en su propio predio y en su gran mayoría poseen título de propiedad. Realizan agricultura 100\% convencional, además de poseer arboles maderables en un $31,58 \%$ y un $10,53 \%$ dedicados al monocultivo. El $63,15 \%$ reportaron rendimientos de cacao entre $200 \mathrm{y}$ $400 \mathrm{~kg} / \mathrm{ha}$ con un $73,68 \%$ de cacaos forasteros, CCN51 y trinitarios. El 42,10\% de fincas diversificaron su producción teniendo entre 7 y 9 cultivos diferentes, $\mathrm{y}$ el $68,42 \%$ de fincas reportaron tener al menos 4 canales de comercialización para sus productos.

El grupo II, representa el 20,51\% de la zona de estudio. Entre sus características resaltan que las fincas cacaoteras poseen como propietarios en partes iguales, tanto a varones como a mujeres. Se reportó que un $87,7 \%$ cuenta con instrucción, de los cuales el 37,5\% tiene más de 61 años que trabajan en su propio predio y que en su gran mayoría poseen título de propiedad. Realizan agricultura $100 \%$ convencional, además de poseer arboles maderables en un $25 \%$ y un $12,5 \%$ dedicados al monocultivo. En donde el 50\% reportaron rendimientos de cacao entre 600 y $800 \mathrm{~kg} / \mathrm{ha}$ con un $50 \%$ de cacaos forasteros, CCN-51 y trinitarios. El 75\% de fincas diversificaron su producción teniendo entre 7 y 9 cultivos diferentes, y el $62,5 \%$ de fincas reportaron tener entre 4 y 5 canales de comercialización para sus productos.

El grupo III, representa el 30,77\% de la zona en estudio. Entre sus características resaltan que las fincas cacaoteras poseen como propietarios un $83,33 \%$ de varones, con $100 \%$ de instrucción, de los cuales el $66,67 \%$ tiene más de 61 años que trabajan en su propio predio y que en su gran mayoría poseen título de propiedad. Realizan agricultura 100\% convencional, además de poseer arboles maderables en un $8,33 \%$ y un $8,33 \%$ dedicados al monocultivo. En donde el $58,33 \%$ reporta rendimientos de cacao entre 600 y 800 $\mathrm{kg} / \mathrm{ha}$ con un $83,33 \%$ de cacaos forasteros, CCN-51 y trinitarios. El 33\% de fincas no diversifico su producción teniendo menos de 3 cultivos diferentes, y el $66,67 \%$ de fincas reportaron tener entre 4 y 5 canales de comercialización para sus productos.

Según Morales et al. (2015), solo entre el 30\% y el 
$40 \%$ de los 20000 a 22000 productores de cacao están organizados en cooperativas. Esto hace, que del $60 \%$ al $70 \%$ de los productores que no están asociados, entreguen sus productos a intermediarios que luego los venden a las grandes empresas exportadoras y empresas locales.

De los 3 grupos mostrados (Figura 2), ninguno de los agricultores pertenece a una asociación. Esta es una de las debilidades del sector agrario, y los efectos que causa esta situación, sobre la comercialización y el financiamiento.

Los grupos I, II y III muestran una extensión menor a 4 hectáreas por productor. La fragmentación de la tierra y la dispersión de parcelas es un gran obstáculo a la rentabilidad del agro. Esto explica gran parte de la baja rentabilidad, debido a su limitación para aprovechar las ventajas de las economías a escala para minimizar costos. Igualmente la atomización del agro no permite una comercialización eficiente, dado los pocos volúmenes producidos de manera dispersa por los productores (POF, 2011). El cacao es un cultivo que requiere de riego permanente, es por eso que los 3 grupos, tienen en su totalidad, acceso al agua.

El grupo I y III cuenta con la totalidad de sus productores con instrucción, mientras que el grupo II el 87,7\% de productores tienen instrucción. El nivel de instrucción entre los encuestados es variable. Existiendo un importante porcentaje de productores agropecuarios en el Perú sin educación $(13,1 \%)$, y la mayor concentración de población en esta condición se encuentra en la sierra, con niveles de analfabetismo que fluctúan entre $14,1 \%$ y $16,7 \%$ (INEI, 2009).

A nivel nacional, los varones representan el 75,5\% del total de productores agropecuarios del país. En la sierra norte, 78,4\% de los agricultores son varones y el resto mujeres (INEI, 2009). En el Grupo I de la zona de Jaén, indica que el 100\% de productores son varones, el Grupo II el 50\% y el Grupo III el 83,33\%, es decir, en promedio, el $77,7 \%$ de los productores de la zona de Jaén son varones.

El grupo I, II y III, muestra que el 52,63\%, el 37,5\%, el $66,67 \%$ respectivamente son productores mayores a
61 años, en promedio, el 62,5\% del os productores en la zona de Jaén son mayores a 61 años. Los productores menores a 61 años, son menos representativos, esto se debe a que los jóvenes tienden a migrar a las ciudades en busca de oportunidades laborales y/o de educación.

La propiedad de la tierra corresponde principalmente a los pequeños agricultores, de los cuales aproximadamente $77 \%$ tiene títulos de propiedad adecuadamente registrados (POF, 2011). Los 3 grupos analizados, muestran que la mayoría de los productores tienen título de propiedad.

Los grupos I y II cuentan con un porcentaje considerable de especies maderables, integradas al sistema agrícola del cacao, contribuyendo de esta manera a controlar la erosión de los suelos ubicados en zonas de alta pendiente. Con ello logran mantener la fertilidad del suelo, ayudando a la sostenibilidad ambiental y el uso adecuado de los recursos naturales (Jiménez, 2002).

Se estima que más de la mitad de las 7 millones de hectáreas de tierras deforestadas al año 2000, se debe a la quema del bosque para labores agrícolas. Estas prácticas de cultivo, ejercen una fuerte presión sobre los bosques primarios y secundarios, generando mayores niveles de deforestación y daños al ecosistema (POF, 2011). Esto se ve reflejado en los grupos de la zona de Jaén, donde los productores acostumbran a quemar la cobertura vegetal del total del área que destinan a sus cultivos, incrementando de esta manera la deforestación y otros daños que esta acarrea, como pérdida de calidad de suelos, entre otras.

Los productores agropecuarios diversifican sus fuentes de ingresos, como estrategia en el manejo de sus riesgos, ante las inclemencias climáticas, pérdida de cosechas o fluctuaciones del mercado. Dentro de esta diversificación, encontramos que su principal fuente, proviene de su propia actividad agropecuaria (INEI, 2009). En la zona de estudio, los grupos I y II diversificaron su producción al ostentar entre 7 y 9 cultivos diferentes, destacando los culticos de arroz, cacao, mango, coco, naranja y palta. Ferro y Meneses (1996) 
señalan que la mayor diversidad de actividades productivas en una finca, aumenta la sostenibilidad económica, convirtiéndoles en sistemas integrales de producción.

En términos porcentuales a nivel nacional, un $44 \%$ de las áreas cultivadas de cacao corresponden al cacao fino (variedades criollo y nativos). Mientras que el cacao común o corriente, conformado por los grupos genéticos CCN-51 (hibrido), corresponde al 53,6\% de los cultivos. El grupo Trinitario y Forastero, suman entre ambos grupos (corrientes) el 56\% del total de cacao sembrado (MINAGRI, 2014). Las características de fincas de las zonas, Jaén nos muestran un alto porcentaje de cacaos forasteros, $\mathrm{CCN}-51$ y trinitarios los cuales son cultivados por su alta capacidad produc- tiva y su resistencia a plagas y enfermedades (MINAGRI, 2016). Sin embargo, esto ocasiona la disminución en las áreas cultivadas de cacao criollo.

La inhabilidad de los pequeños productores para vender sus productos en el mercado, causa una limitante aun teniendo varios canales de comercialización. La primera causa indirecta, se refiere a las malas prácticas de post-cosecha y de transformación de los pequeños productores (POF, 2011). En la zona de Jaén, se tiene un promedio de 4 a más canales de comercialización, pudiendo abrirse más e incluso a mejores canales.

\section{Caracterización zona de Bellavista}

Se logró determinar para esta zona 4 grupos de fincas cacaoteras (Figura 3)

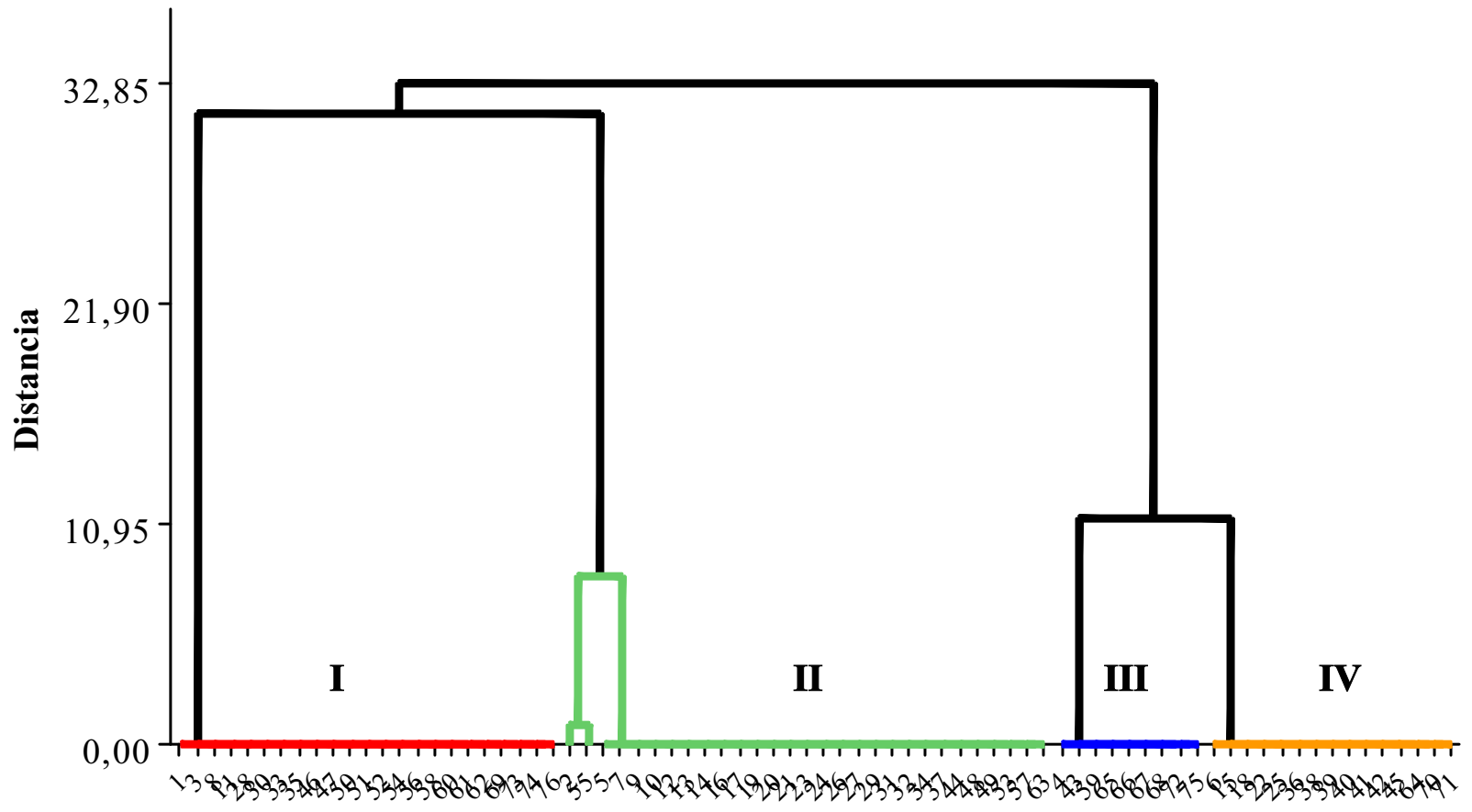

Observaciones

Figura 3. Grupos de fincas cacaoteras para la zona de Bellavista.

Se determinó la existencia de características comunes a los 4 grupos, como que los fundos en su totalidad tienen una extensión inferior a 4 ha, y tienen acceso al agua.

El grupo I, represento el 30,26\% de la zona de estudio. Entre sus características, resaltan que las fincas cacaoteras poseen como propietarios en su totalidad a varones, de los cuales el 13,04\% no cuenta con instrucción, el 47,83\% tiene más de 61 años, el 8,7\% alquila el terreno, reportándose que el $13,04 \%$ de los agricultores no cuenta con el título de propiedad. Solo un 4,35\% realiza agricultura orgánica, además de poseer arboles maderables en un $13,04 \%$ y un $30,44 \%$ dedicados al monocultivo, El 34,78\% reportó rendimientos de cacao menores a $400 \mathrm{~kg} / \mathrm{ha}$ con un $65,22 \%$ de cacaos forasteros, CCN-51 y trinitarios. El 21,73\% de fincas 
no diversifico su producción teniendo, menos de 3 cultivos diferentes y el $60,87 \%$ de fincas reportaron tener al menos 3 canales de comercialización para sus productos.

El grupo II es el mayoritario, representando el 38,16\% de la zona de estudio. Entre sus características, resaltan que las fincas cacaoteras poseen como propietarios en su totalidad a varones con instrucción, de los cuales el 41,38\% tiene más de 61 años, trabajan en su propio predio, con un $24,14 \%$ que pertenece a alguna asociación, donde el 20,69\% no cuenta con título de propiedad. El 3,45\% realiza agricultura orgánica, además de poseer arboles maderables en un 31,03\% y un 44,82\% dedicados al monocultivo. Solo el 20,69\% reporta rendimientos de cacao menores a $400 \mathrm{~kg} / \mathrm{ha}$ con un $68,97 \%$ de cacaos forasteros, $\mathrm{CCN}-51$ y trinitarios. El $34,48 \%$ de fincas no diversifico su producción, teniendo menos de 3 cultivos diferentes y el 55,17\% de fincas reportaron tener más de 5 canales de comercialización para sus productos.

El grupo III, represento el $11,84 \%$ de la zona de estudio. Entre sus características, resaltan que las fincas cacaoteras poseen como propietarios en su totalidad a mujeres, de las cuales el 11,11\% no cuenta con instrucción, el 44,44\% tiene más de 61 años, cultivan en su propio predio y con título de propiedad, donde solo un $11,11 \%$ realiza agricultura orgánica. Poseen arboles maderables en un $22,22 \%$ y un $22,22 \%$ dedicados al monocultivo. El 33,33\% reporto rendimientos de cacao entre 200 y $400 \mathrm{~kg} / \mathrm{ha}$, con un 55,55\% de cacaos forasteros, CCN-51 y trinitarios. El 33,33\% de fincas no diversifico su producción, teniendo menos de 2 cultivos diferentes y el $66,67 \%$ de fincas reportaron tener 4 o más canales de comercialización para sus productos.

El grupo IV, represento el 19,74\% de la zona de estudio. Entre sus características, resaltan que las fincas cacaoteras poseen como propietarios en su totalidad a mujeres, de los cuales el 6,67\% no cuenta con instrucción, el $60 \%$ tiene más de 61 años, cultivan en su propio predio y solo el $26,67 \%$ no cuenta con título de propiedad, donde un $4,35 \%$ realiza agricultura orgáni- ca. Poseen arboles maderables en un $20 \%$ y un $30,44 \%$ dedicados al monocultivo. El 20\% reporto rendimientos de cacao entre 200 y $400 \mathrm{~kg} / \mathrm{ha}$ con un $40 \%$ de cacaos forasteros, CCN-51 y trinitario. El 20\% de fincas no diversifico su producción teniendo menos de 2 cultivos diferentes y el $66,67 \%$ de fincas reporta tener al menos 4 o más canales de comercialización para sus productos.

El grupo II de la zona de Bellavista (Figura 3), tiene un $24,14 \%$ que pertenece a alguna asociación, los demás grupos no presentan productores asociados. Sierra (2012) menciona que las asociaciones de agricultores, han demostrado ser un plataforma poderosa para mejorar los rendimientos y la calidad del cacao, obtener certificaciones ambientales y de otro tipo, y para mejorar la calidad de vida de las comunidades.

Las fincas en su totalidad tienen una extensión menor a 4 hectáreas. Al respecto el INEI (2016) señala que las unidades agropecuarias conducidas por el $32,6 \%$ de pequeños y medianos productores tienen menos de 0,5 hectáreas de superficie agrícola. Por otro lado el 81,8\% de las unidades agropecuarias a nivel nacional tienen un tamaño menor a 5 ha (INEI 2012).

El grupo II cuenta con instrucción y los grupos I, III y IV cuentan con un porcentaje de 13,04\%, 11,11\% y $6,67 \%$ de productores sin instrucción respectivamente. Esto se debe, a que un gran porcentaje de los productores son mayores a 61 años y antiguamente había un difícil acceso a la educación.

El grupo I y II muestra en su totalidad a productores varones y el Grupo III y IV muestra en su totalidad a productoras mujeres. Esto tiene sustento al incremento femenino y a la herencia recibida directamente de sus padres, además, en otros casos, los esposos se dedican a otras actividades y ellas se hacen cargo del cuidado de la finca. En los últimos años, se muestra una mayor presencia femenina y se ha elevado el nivel de educación de los productores, pero ellos también han envejecido (POF, 2011).

Los grupos I, II, III y IV de la zona de Bellavista muestra porcentajes del $47,83 \%, 47,83 \%, 44,44 \%$ y $60 \%$ de productores mayores a 61 años, respectivamente. 
Considerando una distribución por grupos de edad decenal, se observa que a nivel nacional el grupo de adultos comprendidos entre los 35 y 44 años, representa cerca de la cuarta parte de los productores agropecuarios. En tanto que el grupo de edad de jóvenes comprendidos entre los 14 y 24 años, es el grupo minoritario que alcanza menos del $4 \%$ del total. Debido probablemente a la migración del campo a la ciudad por razones de estudio o por cambio de actividad económica. Contrariamente, el grupo de mayor edad comprendido por los productores agropecuarios de la tercera edad de 65 años y más, representan el 20,3\% del total nacional (INEI, 2009).

Los Grupos I, II, III y IV muestra el 13,04\%, 3,45\%, $11,11 \%$ y el $4,35 \%$ respectivamente, a productores que realizan agricultura orgánica, presentando este tipo de agricultura sin aplicaciones técnicas, sino que lo realizan porque el cultivo en algunos casos, poco o nada requiere de productos externos.

En el 2015 el volumen del rendimiento medio era de $720 \mathrm{~kg} / \mathrm{ha}$.La tasa de incremento anual promedio es de 4,4\% entre los años 2009 y 2015 (MINAGRI, 2016). La productividad del cultivo en la zona de Bellavista, muestra un rendimiento promedio menor a $400 \mathrm{~kg} / \mathrm{ha}$ en los grupos analizados. Esta causa puede expresarse por las malas prácticas agrarias de los pequeños productores (generadas por el bajo nivel educativo, falta de acceso a mercados de insumos, falta de información, falta de mercados de créditos y seguros agrarios, entre otros) y la limitada adopción de nuevas tecnologías productivas. En el mediano / largo plazo, surge adicionalmente, una nueva causa indirecta que es el aprovechamiento inadecuado de los recursos naturales, que se genera tanto por causas físicas (naturales), como por malas prácticas agrarias (POF, 2011). Además, las áreas de cultivo no son extensas.

En los 4 grupos, la mayor parte de productores no cuentan con árboles maderables dentro de su predio. Esto es causa de la limpieza total que realizan antes de instalar sus cultivos, lo cual resulta contraproducente para la economía de los agricultores ya que al realizar agroforesteria tendrían un aumento de ingresos ade- más de una disminución de costos cuando existen cultivos asociados (Corven, 1993).

Los productores agropecuarios diversifican sus fuentes de ingresos, como estrategia en el manejo de sus riesgos, ante las inclemencias climáticas, pérdida de cosechas o fluctuaciones del mercado. Dentro de esta diversificación, encontramos que su principal fuente, proviene de su propia actividad agropecuaria (INEI, 2009). En los 4 grupos, hay presencia de 3 cultivos dentro del predio, en algunos grupos hay presencia de monocultivos y en otra mayor diversificación. Generalmente, en las áreas de cultivo de mayor escala, se observa monocultivos, a comparación de las pequeñas áreas de cultivos familiares donde son más diversificadas para proveerse de alimentos.

\section{CONCLUSIONES}

En la zona de Jaén se encontraron 3 grupos, Grupo I (48,72\%), Grupo II (20,51\%), Grupo III (30,77\%). Mientras que la zona de Bellavista se encontraron en 4 grupos, Grupo I (30,26\%), Grupo II (38,16\%), Grupo III (11,84 \%), Grupo IV (19,74 \%). En ambas zonas la extensión de los predios es inferior a 4 ha, contando en su totalidad con acceso al agua

En la zona de Jaén, la totalidad de los agricultores no pertenece a alguna asociación, realizando agricultura convencional, siendo varones la mayoría de sus propietarios, con diversificación de la producción, con rendimientos mayores a $600 \mathrm{~kg} / \mathrm{haen}$ la mayoría de los grupos predominando las variedades forasteras $\mathrm{y}$ trinitarios.

En la zona de Bellavista la mayoría de los agricultores no pertenece a alguna asociación, un porcentaje pequeño realiza agricultura orgánica, siendo varones la mayoría de sus propietarios con poca diversificación de la producción, con rendimientos mayores a $400 \mathrm{~kg} / \mathrm{ha}$ en la mayoría de los grupos predominando las variedades forasteras y trinitarios.

\section{V.AGRADECIMIENTOS}

Al Círculo de Investigación para la Innovación y el Fortalecimiento de la Cadena de Valor del Cacao Nati- 
vo Fino de Aroma en la Zona Nor Oriental del Perú

(CINCACAO-FONDECYT), por el financiamiento.

\section{REFERENCIAS BIBLIOGRÁFICAS}

Altieri, M. A., M. F. Funes, P. Petersen, T. Tomic, y C. Medina. 2011. "Sistemas agrícolas ecológicamente eficientes para los pequeños agricultores". En III Foro Europeo de Desarrollo Rural.Palencia (España).

Anzules, V., R. Borjas, V. Castro, y A. Julca. 2018. "Caracterización de fincas productoras de cacao (Theobroma cacao L.) en Santo Domingo de Los Tsáchilas, Ecuador.” Bosques Latitud Cero 8 (2): 39-50.

Cabrera, D., A. García, R. Acero, A. Castaldo, J. Perea, y J. Martos. 2004. Metodología para la caracterización y tipificación de sistemas ganaderos. Documentos de trabajo Producción Animaly Gestión. Córdoba (España).

Casas, G. y N. Veitía. 2008. “Aplicación de métodos de comparaciones múltiples en biotecnología vegetal."Biotecnología Vegetal 8(2): 67-71.

Collantes, R., A. Rodríguez, y M. Canto. 2017. “Caracterización de fincas productoras de palto (Persea americana Mill.) y mandarina (Citrus Spp.) en Cañete, Lima, Perú.” Aporte Santiaguino 8 (1): 33-44.

Corven, J. 1993. “Asociación de cultivos con cacao, aspectos económicos.” En I Seminario Regional "Sombras y cultivos asociados con cacao". Turrialba (Costa Rica).

FAO (Organización de las Naciones Unidas para la Alimentación y la Agricultura) y USAC (Universidad de San Carlos de Guatemala). 1995. Caracterización del sistema agrario que comprende la zona de retornados "Nueva Esperanza”, Nentón, Huehuetenango. Informe de Proyecto. Guatemala (Guatemala).

Ferro, J. y Meneses, O. 1996. "Los productores de cacao en el municipio de Arauquita, departamento de Arauca, Colombia". Cuadernos de Desarrollo Rural 37: 125-137.
Guerrero, M. 2007. Diagnóstico y propuesta de parámetros para la estandarización y homogenización del tratamiento poscosecha de cacao. Lima (Perú): Ministerio de Economía y Finanzas.

INEI (Instituto Nacional de Estadística e Informática). 2009. Perú: Perfil del productor agropecuario. Lima (Perú): INEI.

INEI (Instituto Nacional de Estadística e Informática). 2012. Resultados Definitivos IV Censo nacional agropecuario - 2012. Lima (Perú): INEI.

INEI (Instituto Nacional de Estadística e Informática). 2016. Encuesta nacional agropecuaria: Principales resultados, pequeñas medianas y grandes unidades agropecuarias. Lima (Perú): INEI

Jiménez, F. 2002. Metodología rápida para estimar el manejo de una microcuenca. Turrialba (Costa Rica): CATIE.

León-Velarde, C. U y R. G. Quiroz. 1994. Análisis de sistemas agropecuarios; uso de métodos biomatemáticos. La Paz (Bolivia): Centro de Investigación de Recursos Naturales y Medio Ambiente - Consorcio para el Desarrollo Sostenible de la Ecorregión Andina.

López, F., R. Gómez, C. Harvey, M. López, y F. Sinclair. 2007. "Toma de Decisiones de Productores Ganaderos Sobre El Manejo de Los Árboles En Potreros de Matiguás, Nicaragua." Agroforestería En Las Américas 45: 93-99.

Macedo, R., M. A. Gallina, J. M. Zorrilla, J. M. Palma, y J. Pérez. 2003. “Análisis de un sistema de producción tradicional En Colima, México.” Archivos de Zootecnia 52 (200): 463-74.

Malagon, R. y M. Praguer. 2001. El enfoque de sistemas: Una opción para el análisis de las unidades de producción agrícola. Palmira (Colombia): Universidad Nacional de Colombia.

Merma, I. y A. Julca. 2012. “Caracterización y evaluación de la sustentabilidad de fincas en Alto Urubamba, Cusco, Perú.” Ecología Aplicada 


$$
11(1): 1-11 .
$$

MINAGRI (Ministerio de Agricultura y Riego). 2014.

Catálogo de Cultivares de Cacao del Perú. Lima (Perú): MINAGRI.

MINAGRI (Ministerio de Agricultura y Riego). 2016. Estudio del Cacao en el Perú y el Mundo: Un análisis de la producción y el comercio. Lima (Perú): MINAGRI.

Morales, O., A. Borda, A. Argandoña, R. Farach, L. G. Naranjo, K. Lazo. 2015. La Alianza Cacao Perú y la cadena productiva del cacao fino de aroma. Lima (Perú): esan ediciones.

POF (Peru Opportunity Fund). 2011. Diagnóstico de la Agricultura en el Perú. Informe Final. Lima (Perú).

Saldaña, P. 2019. La producción de cacao peruano a um entaria en 2019. https://mercadosyregiones.com/2019/05/29/1 a-produccion-de-cacao-peruano-aumentariaen-2019/ (Consultada 10 de julio de 2020).

Santistevan, M., A. Julca, R. Borjas, y O. Tuesta. 2014. "caracterización de fincas cafetaleras en la localidad de Jipijapa (Manabí, Ecuador).” Ecología Aplicada 13(2): 187-192.

Scheaffer, R., W. Mendenhal, y L. Ott. 1987. Elementos de muestreo. México DF (México): Editorial Iberoamericano.

Sierra, A. Y. 2012. “APROCAFRUM: Una crónica sobre el cacao, el alimento de la paz y el bienestar.” En Sostenibilidad y Legalidad: Buenas prácticas ambientales en los programas de desarrollo alternativo. M. Chavarro, S. C. Torres, y H. F. Santos (eds). Bogotá (Colombia): UACT y UNODC

Tuesta, O., A. Julca, R. Borjas, P. Rodríguez, y M. Santistevan. 2014. "Tipología de fincas cacaoteras en la subcuenca media del Río Huayabamba, Distrito de Huicungo (San Martín, Perú).” Ecología Aplicada 13 (2): 7178.

Vargas, B., O. Solís, F. Sáenz, y H. León. 2013. “Caracterización y clasificación de hatos leche- ros." Agronomía Mesoamericana 24 (2): 257-75. 